\title{
Relevância e tratamento das lesões características da insuficiência cardíaca na musculatura esquelética
}

\section{Relevance and treatment of heart failure's injuries to skeletal musculature}

Carlos R. Bueno Júnior

\begin{abstract}
RESUMO
Pacientes com insuficiência cardíaca, a via final das doenças cardiovasculares e a primeira causa de internação em idosos no Brasil, apresentam deterioração do tecido muscular esquelético. Além disso, as lesões na musculatura têm relação direta com a qualidade de vida e a autonomia dos pacientes, por meio do nível de tolerância aos esforços físicos, além de constituírem um preditor independente de mortalidade na síndrome.

Ao longo da presente revisão de literatura serão discutidos em relação à insuficiência cardíaca: seu conceito e um breve histórico; a importância da musculatura esquelética; as anormalidades funcionais, estruturais, bioquímicos e moleculares que acometem esse tecido; as potenciais causas dessas alterações (hiperatividade simpática, redução na ingestão alimentar, espécies reativas de oxigênio, citocinas pró-inflamatórias e diminuição do nível de atividade física e do fluxo sanguíneo); e os efeitos da principal terapia direcionada para combater essa disfunção, o treinamento físico aeróbio de intensidade moderada.

Reconhecida a importância do tecido muscular esquelético na síndrome, essa é uma área da investigação científica com muitos temas ainda não estudados, como o efeito de terapias farmacológicas utilizadas pelos pacientes, de exercícios físicos aeróbios de maior intensidade e intervalados, de exercícios físicos de força, de intervenções nutricionais e de terapias celulares e gênicas. No futuro, os resultados desses estudos que serão desenvolvidos certamente contribuirão para uma menor degeneração da musculatura esquelética na doença, o que, consequentemente, vai melhorar a qualidade de vida, a autonomia e o prognóstico dos pacientes.
\end{abstract}

Palavras-chave: Insuficiência Cardíaca. Sistema Musculoesquelético. Biologia Molecular. Terapia.

Mestre pela Escola de Educação Física e Esporte da Universidade de São Paulo
Correspondência:

Carlos Roberto Bueno Júnior Escola de Educação Física e Esporte - USP

Av. Prof. Mello Morares, 65 05508-900 - São Paulo - SP - BRASIL Endereço eletrônico: buenojr@usp.br

Artigo recebido em 05/07/2009 Aprovado em 29/09/2009 


\section{Introdução}

Os avanços científicos e tecnológicos na área da saúde, aliados ao estilo de vida preponderante da sociedade moderna, no qual o sedentarismo, o fumo, a obesidade, as dislipidemias, a diabetes mellitus e a hipertensão arterial são frequentes, geraram uma inversão epidemiológica. Doenças infecciosas e parasitárias, que no passado eram responsáveis por grande parte da mortalidade em todo o mundo, passassem a ser gradativamente substituídas pelas doenças crônicodegenerativas, que cada vez mais se apresentam como um grande desafio à humanidade. Segundo Prata $(1989)^{1}$, por exemplo, enquanto em 1930 as doenças infecciosas e parasitárias eram responsáveis por $46 \%$ e as doenças do aparelho circulatório por $12 \%$ dos óbitos no Brasil, em 1985 esses números foram 7\% e $33 \%$, respectivamente.

As doenças cardiovasculares, que são crônicodegenerativas, são a principal causa de morte no mundo, sendo responsáveis por cerca de $30 \%$ de todos os óbitos e têm a insuficiência cardíaca como a síndrome final da maioria delas. Um dado que é representativo da magnitude de importância da insuficiência cardíaca em nossa nação é que ela é a terceira causa de internação geral no Brasil e a primeira em pessoas com mais de 65 anos de idade, sendo responsável por grande montante dos gastos com saúde no país - $\mathrm{R} \$$ $4.033,62$ por ano para cada paciente internado, segundo o exemplo de Araújo et al. (2005) ${ }^{2}$.

\section{Insuficiência cardíaca: definição e breve histórico}

Uma das definições mais antigas de insuficiência cardíaca é a de Thomas Lewis, de 1933, segundo a qual a síndrome é uma condição na qual o coração falha em descarregar seus conteúdos adequadamen$t^{3}$. Atualmente essa síndrome clínica é caracterizada por: falência no suprimento adequado de sangue em relação ao retorno venoso e às necessidades metabólicas teciduais ${ }^{4}$; mau prognóstico, fadiga, dispnéia e grande limitação a esforços físicos ${ }^{5,6}$; remodelamento cardíaco (hipertrofia, dilatação da câmara, apoptose e aumento na fração de colágeno), disfunção endotelial, hiperatividade simpática, retenção de fluido e exacerbação da atividade dos sistemas reninaangiotensina-aldosterona e argininina-vasopressina ${ }^{7} ; \mathrm{e}$ alterações no perfil metabólico e morfológico dos músculos esqueléticos ${ }^{8,9}$ (Figura 1 ).

Descrições da insuficiência cardíaca existiam nas sociedades antigas egípcia, grega e índica, e os romanos antigos usavam a planta foxglove como medicamento para tal síndrome. Pouco entendimento da natureza desta condição podia existir até que William Harvey descreveu a circulação sanguínea em 1628. A descoberta de Wilhelm Röntgen do raio-X em 1895 e o desenvolvimento da eletrocardiografia também na década de 1890 possibilitaram avanços na investigação da síndrome. O advento da ecocardiografia, da cateterização cardíaca e da medicina nuclear tem

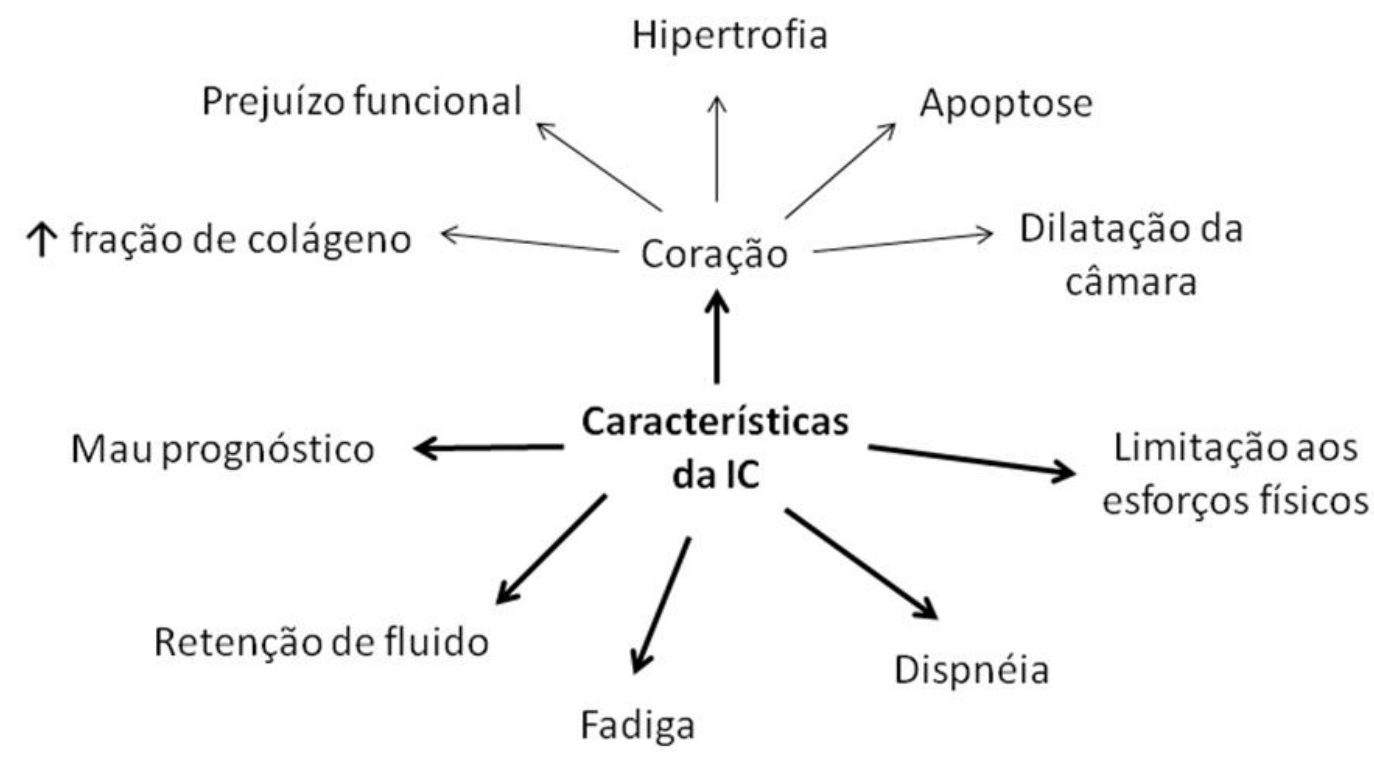

Figura 1: Características gerais da insuficiência cardíaca (IC) 
melhorado o diagnóstico e o tratamento dos pacientes com insuficiência cardíaca. Sangrias e sanguessugas foram utilizadas por séculos no tratamento da síndrome e William Withering publicou suas considerações dos benefícios dos medicamentos digitálicos em 1785 No século XIX e início do século XX, a insuficiência cardíaca associada com a retenção de fluido era tratada com os tubos de Southey, que eram inseridos nos edemas periféricos, permitindo alguma drenagem. Os diuréticos com mercúrio, usados desde 1920, foram associados a uma toxicidade substancial, improvável nos diuréticos tiazídicos, que foram introduzidos em 1958. Vasodilatadores não eram amplamente utilizados até o desenvolvimento dos inibidores da enzima conversora de angiotensina na década de 1970. Outro marco foi o estudo CONSENSUS-1, o primeiro estudo cooperativo na Escandinávia do Norte, publicado em 1987, que mostrou benefícios inequívocos de sobrevivência com o medicamento enalapril em pacientes com insuficiência cardíaca. Finalmente em 1995 a Sociedade Européia de Cardiologia publicou um guia para o diagnóstico da doença. ${ }^{3}$

\section{Importância da musculatura esque- lética na insuficiência cardíaca}

A relevância da musculatura esquelética na insuficiência cardíaca surgiu há apenas alguns anos e isso tem relação com o desenvolvimento de dois conceitos relacionados à importância da musculatura esquelética na síndrome.

O primeiro conceito é que a caquexia (do grego: kakos, má, ruim e hexis, condição do corpo) é um preditor independente de mortalidade na insuficiência cardíaca. ${ }^{10}$ A caquexia é uma condição resultante de várias doenças sistêmicas como o câncer, a síndrome da imunodeficiência adquirida (AIDS), o diabetes mellitus, a sepsis e a insuficiência renal. ${ }^{11,12}$ Pacientes portadores de insuficiência cardíaca que manifestam o quadro de caquexia normalmente apresentam perda excessiva de massa corporal associada à perda de massa óssea, gorda e muscular, além de apresentarem mortalidade aumentada. ${ }^{10} \mathrm{O}$ mecanismo pelo qual essa redução na massa muscular esquelética eleva os índices de mortalidade, no entanto, não é conhecido. Segundo Anker et al. ${ }^{13}$, por exemplo, a área média do quadríceps de pacientes com insuficiência cardíaca caquéticos foi $45,9 \mathrm{~cm}^{2}$, contra $61,6 \mathrm{~cm}^{2}$ dos não caquéticos.

O segundo conceito relacionado à importância da musculatura esquelética na insuficiência cardíaca é que a intolerância aos esforços físicos, uma das principais características da síndrome, usada como critério para classificações do nível de gravidade da síndrome e muitas vezes o sintoma limitante, não é uma consequência da disfunção cardíaca. Foi evidenciado que a correlação entre o nível de prejuízo nas variáveis cardíacas e o grau de tal intolerância é baixa. A partir desses achados, mudanças intrínsecas à musculatura esquelética começaram a ser investigadas, e foi demonstrado que há correlação entre o nível dessa intolerância aos esforços físicos e o grau de prejuízo funcional da musculatura esquelética. ${ }^{14-17}$ Além disso, o aumento no débito cardíaco agudo provocado por drogas não melhora a tolerância aos esforços físicos na insuficiência cardíaca. ${ }^{18,19}$ Por fim, Jondeau et al. $(1992)^{20}$ mostraram em um estudo bem delineado que as lesões periféricas, e não a redução no débito cardíaco, limitam a maioria dos pacientes com insuficiência cardíaca. Eles viram que a frequência cardíaca e o consumo de oxigênio aumentaram na maioria dos pacientes adicionando um exercício com os membros superiores após o consumo de oxigênio ter atingido platô em exercício em bicicleta até a exaustão.

Portanto, além de aumentar os índices de mortalidade, a deterioração do tecido contrátil esquelético reduz a autonomia e a qualidade de vida dos pacientes com insuficiência cardíaca por meio do aumento na intolerância aos esforços físicos. ${ }^{21}$

\section{Lesões na musculatura esquelética características da insuficiência car- díaca}

As anormalidades do tecido muscular esquelético na insuficiência cardíaca serão descritas em quatro tópicos: alterações funcionais, alterações estruturais, alterações bioquímicas e alterações moleculares.

\section{Alterações funcionais}

A redução na capacidade de gerar tensão é encontrada tanto em modelos experimentais quanto em pacientes com insuficiência cardíaca, esse prejuízo é verificado tanto na força máxima como na resistência à fadiga ${ }^{22,23}$, e suas causas são os prejuízos estruturais, bioquímicos e moleculares descritos abaixo.

\section{Alterações estruturais}

As alterações estruturais mais características do tecido muscular esquelético na insuficiência cardíaca são a alteração na proporção dos diferentes tipos 
de fibras musculares, a morte celular programada de miócitos e a atrofia. ${ }^{22,23}$ Em relação à primeira alteração, sabe-se que na insuficiência cardíaca há uma redução na proporção de fibras tipo I (mais oxidativas e resistentes à fadiga) e consequente aumento no percentual de fibras tipo II (mais glicolíticas e menos resistentes à fadiga). ${ }^{23}$

Em relação à atrofia muscular na insuficiência cardíaca, por sua vez, vários estudos estão sendo desenvolvidos no sentido de entender suas causas e inibi-la. ${ }^{24,25}$ Até o momento é conhecido que ela é gerada por um desequilíbrio entre vias moleculares de síntese e de degradação protéica ${ }^{26}$, que serão descritas posteriormente.

\section{Alterações bioquímicas}

Diminuição da atividade de enzimas oxidativas, do número e do volume de mitocôndrias, rarefação capilar, acidose precoce, acúmulo excessivo de lactato, e depleção mais rápida de fosfatos de alta energia são aspectos marcantes no tecido muscular esquelético na insuficiência cardíaca. ${ }^{22,23}$ Todas essas características estão relacionadas à transição de fibras mais oxidativas para mais glicolíticas, descrita acima, e têm como consequência a antecipação da fadiga. ${ }^{22}$

Os principais prejuízos característicos da musculatura esquelética na insuficiência cardíaca apresentados até aqui estão representados na Figura 2.

\section{Alterações moleculares}

Em relação às anormalidades moleculares, descreveremos aspectos relacionados ao controle do transporte do $\mathrm{Ca} 2+$ intracelular e às vias de síntese e degradação protéica (Figura 3).

Cálcio. A descoberta de que a habilidade dos músculos de contrair depende da presença de $\mathrm{Ca}^{2+}$ ocorreu em 1882, quando Ringer percebeu que o coração de rã contraía quando era incubado em solução preparada com água de torneira de Londres, mas não quando preparada com água destilada. ${ }^{27}$ Muito tempo se passou e em 1993 chegou-se à conclusão que a diminuição na produção de força máxima dos pacientes com insuficiência cardíaca persiste mesmo quando as forças são normalizadas pela massa muscular úmida e/ou pela área de secção transversa do músculo. ${ }^{28}$ Além disso, as anormalidades supracitadas, como atrofia, mudança do tipo de fibra e menor prevalência do metabolismo aeróbio, não são prováveis de explicar completamente o padrão de fadiga muscular dos pacientes, que devido à sua velocidade e gravidade parece refletir prejuízo no acoplamento excitação-contração ${ }^{16,29-31}$, que está diretamente relacionado ao íon $\mathrm{Ca}^{2+}$.

Após Perreault et al. (1993) ${ }^{28}$ encontrarem prejuízo no transiente de $\mathrm{Ca}^{2+}$ na insuficiência cardíaca na musculatura esquelética em 1993, começaram a ser estudadas a expressão das proteínas que transportam esse íon em diferentes etiologias e níveis de gravidade de insuficiência cardíaca, porém os estudos ainda são muito escassos. O trabalho supracitado foi o primeiro a relatar redução na magnitude e na velocidade da captação de $\mathrm{Ca}^{2+}$ pelo retículo sarcoplasmático após a contração em associação com redução na tensão e na força tetânica no músculo extensor longo dos dedos de ratos com insuficiência cardíaca. Por

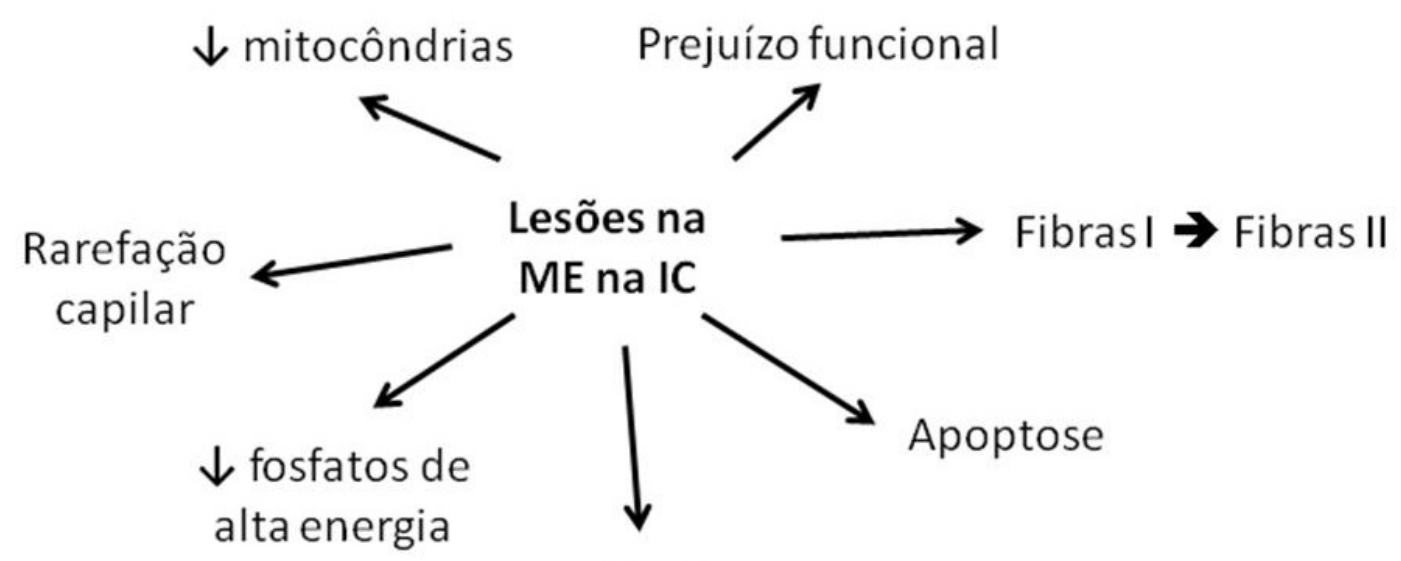

Atrofia

Figura 2: Principais lesões na musculatura esquelética (ME) em pacientes com insuficiência cardíaca (IC). 
outro lado, um estudo publicado em 1999 foi o primeiro a registrar que a expressão gênica da isoforma de sarco endo-retículo $\mathrm{Ca}^{2+}$-ATPase 2 (SERCA 2) estava reduzida no músculo sóleo de ratos infartados com insuficiência cardíaca. ${ }^{32}$ Contrário a esses achados, Lunde et al. (2001) $)^{33}$ não encontraram diferenças na expressão de isoformas de SERCA, de receptor para rianodina $(\mathrm{RR})$ e de diferentes subunidades do trocador $\mathrm{Na}^{+}-\mathrm{Ca}^{2+}(\mathrm{NCX})$ utilizando os músculos sóleo e flexor curto dos dedos de ratos controle e ratos com insuficiência cardíaca induzida por ligadura da artéria coronária esquerda. Os resultados controversos do efeito da insuficiência cardíaca na expressão de proteínas envolvidas no transiente de $\mathrm{Ca}^{2+}$ podem ser explicados por níveis de gravidade e etiologias de insuficiência cardíaca distintos, diferentes espécies estudadas, músculos utilizados distintos e/ou diferentes métodos de análise.

Além do $\mathrm{Ca}^{2+}$ estar diretamente envolvido na contração e no relaxamento, a alterações de suas concentrações pode estimular processos deletérios à musculatura esquelética, como atrofia, apoptose e diminuição da capacidade regenerativa. ${ }^{34}$

Vias de síntese e degradação protéica. A atrofia muscular, observada na insuficiência cardíaca, ocorre devido a uma redução na atividade das vias de síntese e/ou aumento na atividade das vias de degradação protéica. Na musculatura esquelética duas vias de degradação são consideradas as mais importantes: as calpaínas e o sistema ubiquitina-proteossoma.
As calpaínas são proteases ativadas por concentrações aumentadas de $\mathrm{Ca}^{2+}$ no citosol, que clivam proteínas contráteis e estruturais em peptídeos. Um regulador importante desse sistema são as calpastatinas, que são proteínas capazes de inibir as calpaínas. $\mathrm{Na}$ insuficiência cardíaca, o prejuízo nas proteínas relacionadas ao transporte intracelular de $\mathrm{Ca}^{2+}$, supracitado, poderia ter como consequência uma exacerbação das concentrações desse íon capaz de ativar essas proteases. ${ }^{35}$

Já o sistema ubiquitina-proteossoma tem como princípio a degradação de peptídeos, muitas vezes provenientes da clivagem pelas calpaínas, "etiquetados" com ubiquitinas. Essa ubiquitinação é principalmente regulada por enzimas chamadas E3 ligases, que catalisam a transferência de ubiquitinas ativadas e conjugadas em um complexo de poliubiquitinas para a proteína-alvo, que é então reconhecida pelo proteossoma 26S (organela do citoplasma com $\mathrm{pH}$ ácido) e degradada em pequenos peptídeos. Dentre as E3 ligases, destacam-se a Atrogin-1/ MAFbx, MuRF1 e E3a, que são altamente expressas na musculatura esquelética e aumentam consideravelmente sua expressão em estados de atrofia, como jejum, sepsis, câncer, diabetes e AIDS. ${ }^{36-39}$ Schulze e colaboradores recentemente (2005) demonstraram que a E3 ligase Atrogin-1/MAFbx está com sua expressão aumentada no tecido muscular esquelético de camundongos com disfunção ventricular decorrente de infarto do miocárdio.

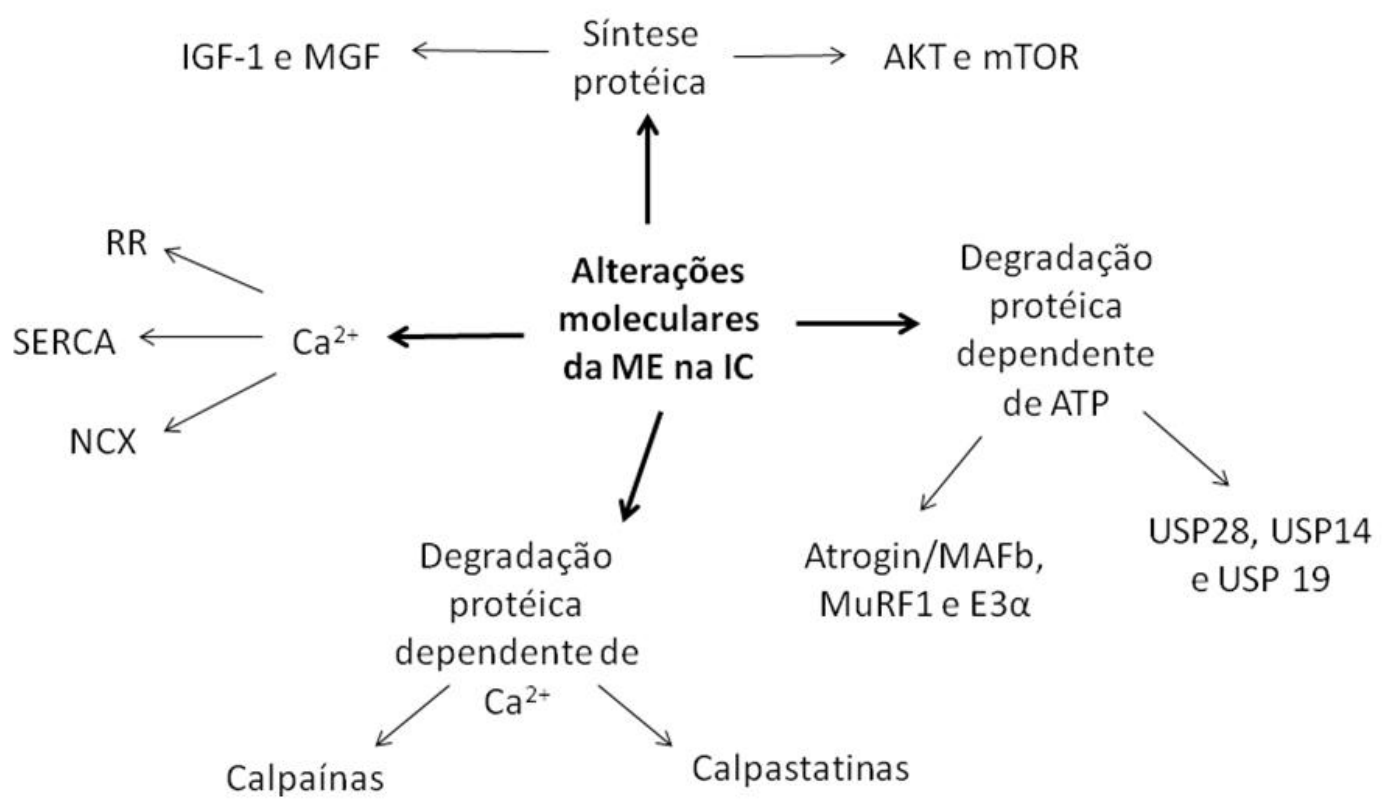

Figura 3: Alterações moleculares que acometem a musculatura esquelética (ME) na insuficiência cardíaca (IC). 
Outra classe de proteínas do sistema ubiquitinaproteossoma são as deubiquitinases, como a USP28, a USP14 e a USP19, que reconhecidamente são altamente expressas na musculatura esquelética e regulam o processo de ubiquitinação por meio da remoção das ubiquitinas dos substratos protéicos marcados para a degradação. Portanto, as proteínas deubiquitinadas deixam de ser conduzidas à degradação protéica. $\mathrm{O}$ papel das deubiquitinases na insuficiência cardíaca, no entanto, não é conhecido. ${ }^{37}$

Outro potente regulador trófico do tecido muscular esquelético é o IGF-1, que pode acionar vias de síntese e inibir vias de degradação protéica. Sabe-se que os músculos atrofiados de animais com insuficiência cardíaca possuem redução tanto nas concentrações de IGF-1 circulante, quanto na expressão de IGF1 muscular (MGF; Mechano Growth Factor). Portanto, a manipulação dos níveis de IGF-1 ou as vias ativadas por este peptídeo é uma estratégia atraente no combate à atrofia muscular em quadros de insuficiência cardíaca. ${ }^{40}$

\section{Causas das lesões na musculatura esquelética na insuficiência cardíaca}

Vários fatores, que serão discutidos a seguir, são potenciais candidatos por deflagrarem todos es- ses prejuízos que foram descritos na musculatura esquelética na insuficiência cardíaca (Figura 4). O entendimento deles, por sua vez, é uma ferramenta importante para o desenvolvimento de estratégias terapêuticas eficientes.

\section{Hiperatividade simpática}

Acredita-se que a hiperatividade simpática contribui para a deterioração na musculatura esquelética na insuficiência cardíaca pela alteração do turnover protéico, ou seja, um desequilíbrio entre a síntese e a degradação protéica que favoreceria a perda de massa muscular. No entanto, recentemente foi demonstrado que o tratamento com clembuterol (agonista $\beta 2$-adrenérgico) reduziu a atrofia por desuso por meio da diminuição dos componentes do sistema ubiquitina-proteossoma. ${ }^{41}$ Nesse estudo não foi observada alteração do peptídeo IGF-I, demonstrando que a ação da ativação via receptores $\beta$-adrenérgicos tem como principal efeito a inibição da degradação protéica e não a ativação da síntese protéica. Outros estudos atribuem esse efeito anabólico devido à inibição no sistema proteolítico dependente de $\mathrm{Ca}^{2+},{ }^{42,43}$ de modo que os mecanismos moleculares envolvidos no ganho de massa muscular ainda não estão bem esclarecidos. Portanto, a administração de agonistas $\beta 2$-adrenérgicos em curto prazo favorece a prevenção da perda de massa muscular, que parece ocorrer por atenuação da degradação muscular.

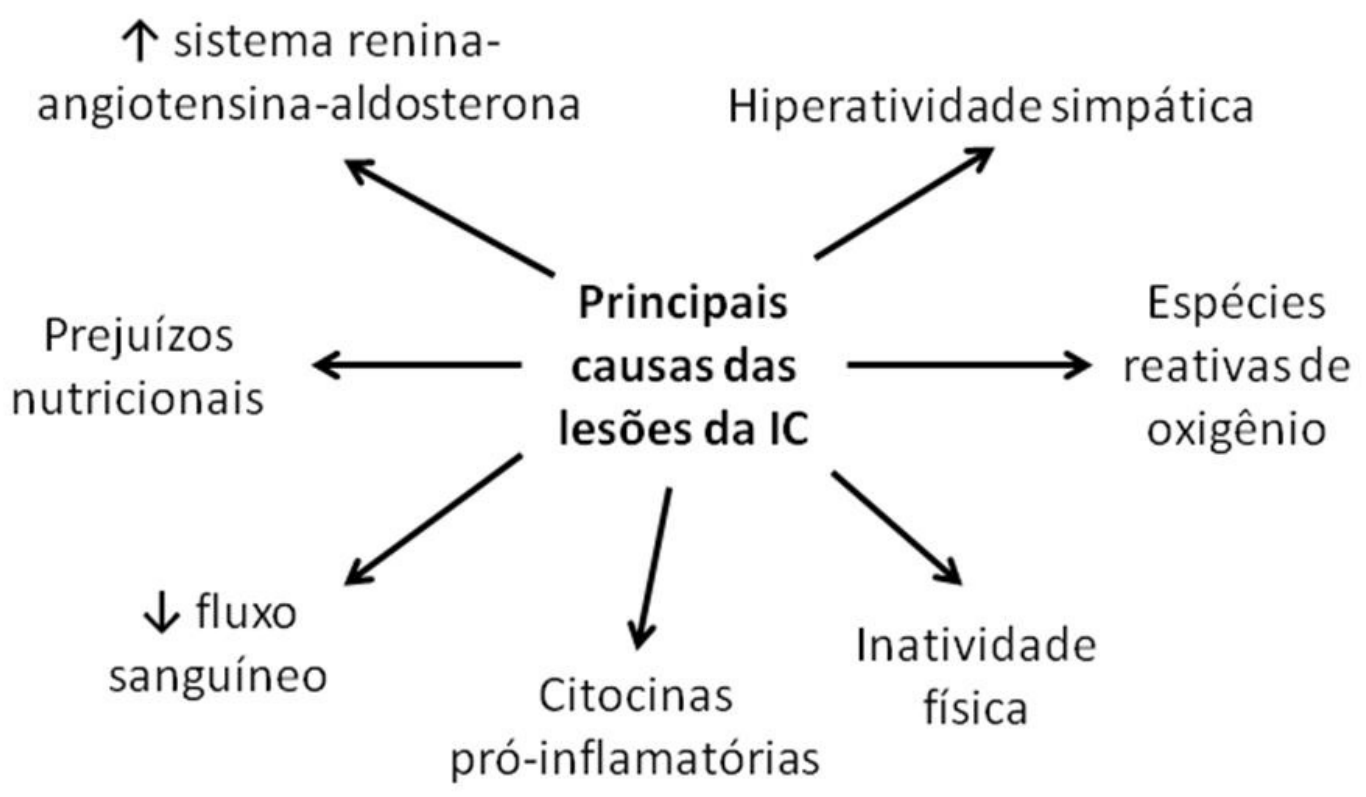

Figura 4: Principais causas das lesões causadas ao organismo que são características da insuficiência cardíaca (IC). 
O efeito promotor de crescimento atribuído aos agonistas $\beta 2$-adrenégicos, por sua vez, é limitado pelo tempo de administração da droga, apresentando atenuação do efeito hipertrófico em tratamentos prolongados. ${ }^{44-46}$ Essa desaceleração da taxa de crescimento ocorre pela redução da densidade de receptores $\beta$-adrenérgicos, causada pela exposição crônica dos receptores ao agonista. Um estudo demonstrou que o tratamento com clembuterol por 18 dias reduziu $50 \%$ da densidade de receptores $\beta 2$-adrenérgicos na musculatura esquelética. ${ }^{47}$ De fato, o primeiro estudo de internalização de receptor $\beta 2$-adrenérgico demonstrou que após 30 minutos de estimulação por agonista $\beta 2$-adrenérgico, cerca de $50 \%$ dos receptores do músculo sóleo foram internalizados e somente $30 \%$ retornaram a superfície celular. ${ }^{48}$ Portanto, cronicamente a hiperatividade simpática levaria à internalização dos receptores $\beta$-adrenérgicos, o que levaria à degradação protéica da musculatura esquelética.

Outra hipótese é que a hiperatividade simpática contribui para a vasoconstrição crônica dos pacientes tanto no repouso como durante a atividade física no tecido muscular esquelético, o que favoreceria uma redução no fluxo sanguíneo e, consequentemente, a disfunção muscular. ${ }^{49}$

\section{Redução na ingestão alimentar}

Apesar do reconhecimento de que a nutrição inadequada é uma complicação da síndrome desde o tempo de Hipócrates ${ }^{50}$, essa nutrição inadequada atualmente ainda permanece como um grande problema de saúde..$^{51}$

A redução na ingestão calórica e protéica, aliada à má absorção intestinal, que são características da insuficiência cardíaca, pode ter como consequência uma menor eficiência no suprimento de energia e aminoácidos à musculatura esquelética, o que pode favorecer a redução na síntese protéica e a antecipação da fadiga. ${ }^{52}$

Além disso, esse quadro pode levar a uma série de alterações hormonais no sentido do organismo "economizar" energia, como a redução nas concentrações do hormônio tireoidiano, que entre inúmeras consequências, é capaz de reduzir a expressão de proteínas envolvidas no transporte intracelular de $\mathrm{Ca}^{2+}$, deteriorando ainda mais o tecido muscular esquelético. ${ }^{53}$

\section{Estresse oxidativo}

É conhecido que as espécies reativas de oxigênio são produzidas na musculatura esquelética tanto em repouso quanto durante a contração muscular, atuando como segundos mensageiros nas vias de sinalização celular que regulam tanto processos fisiológicos como patológicos. ${ }^{54}$ Além disso, alguns estudos mostraram um aumento na produção de espécies reativas de oxigênio e em marcadores de estresse oxidativo em modelos experimentais de atrofia muscular, ${ }^{55,56}$ ao passo que a degradação muscular parece ser prevenida pela administração de antioxidantes exógenos. ${ }^{57,58}$

Até o momento não é conhecido como as espécies reativas de oxigênio contribuem para a deterioração muscular na insuficiência cardíaca, porém tem sido sugerido que a ativação do fator nuclear kappa B (NF-кB), que é um fator de transcrição redox sensível capaz de aumentar a expressão de importantes componentes do sistema ubiquitina-proteossoma, seria importante nesse processo. ${ }^{59,60}$

Além dos estudos em modelos experimentais de insuficiência cardíaca, já é bem documentado na literatura científica que pacientes com a síndrome apresentam um aumento exacerbado e prejudicial na concentração de espécies reativas de oxigênio no tecido contrátil apendicular e que o exercício físico, que será discutido posteriormente, reduz essas concentrações. ${ }^{61,62}$

\section{Citocinas pró-inflamatórias}

Tanto a inflamação sistêmica como a local têm sido sugeridas ter papel importante na patogênese e progressão da insuficiência cardíaca. ${ }^{63}$ As citocinas, como o fator de necrose tumoral $\alpha$ (TNF $\alpha)$ e a interleucina 1 (IL-1), podem afetar o metabolismo muscular e a geração de força por efeitos diretos, por exemplo, por alterar a expressão da $\mathrm{Ca}^{2+}$-ATPase que bombeia o $\mathrm{Ca}^{2+}$ para dentro do retículo sarcoplasmático e seu regulador fosfolambam, ${ }^{64}$ ou por induzir outros fatores patológicos, como o aumento na expressão de óxido nítrico sintase induzível (iNOS). ${ }^{65,66}$

Já foi mostrado que tanto a expressão dessas citocinas como a expressão de iNOS estão aumentadas na musculatura esquelética de pacientes com insuficiência cardíaca. Além disso, tem sido proposto que um nível excessivo de óxido nítrico intracelular produzido pela iNOS pode inibir enzimas aeróbicas chave, como a citocromo c oxidase da cadeia de transporte de elétrons e, consequentemente, diminuir o consumo de oxigênio de pico. ${ }^{67}$

A observação de que a expressão de citocinas pró-inflamatórias ocorre na ausência de infiltração de 
monócitos ou macrófagos indica que os miócitos esqueléticos podem produzir essas citocinas de modo parácrino e/ou autócrino em quantidades suficientes para induzir a expressão de iNOS.$^{67}$ A expressão de iNOS pode ser induzida por três fatores patogênicos conhecidos: citocinas (interleucina $1-\beta$, TNF- $\alpha$ na presença de interleucina $1-\beta$ ), espécies reativas de oxigênio e fatores mecânicos, como aumentado estresse na parede do miocárdio. ${ }^{68}$

Além disso, tem sido proposto que na musculatura esquelética de pacientes com insuficiência cardíaca, o acúmulo de óxido nítrico produzido pelo aumento da expressão de iNOS reduz a expressão de creatina quinase mitocondrial, uma enzima chave para a transferência de fosfatos de alta energia da fosforilação oxidativa mitocondrial para os filamentos de miosina do citoplasma. Isso pode explicar, pelo menos em parte, a depleção aumentada de fosfocreatina, que ocorre devido a uma ressíntese de fosfocreatina mitocondrial reduzida. ${ }^{69}$

Por fim, o fator de necrose tumoral $\alpha$ (TNF $\alpha)$ e a interleucina 1 (IL-1) também contribuem para o aumento da degradação protéica e a redução na ativação de vias de síntese pelo fator de crescimento similar à insulina (IGF-1). ${ }^{11,70}$

\section{Diminuição do nível de atividade física}

Várias das anormalidades características da musculatura esquelética na insuficiência cardíaca, como rarefação capilar, atrofia e fadiga precoce, reconhecidamente estão presentes em indivíduos que reduzem o nível de atividade física, um processo geralmente conhecido como destreinamento. O que ocorre com os pacientes da síndrome é que alguns fatores, como a dispnéia e a fadiga precoce, tendem a torná-los menos ativos fisicamente, o que deteriora ainda mais o tecido muscular esquelético, criando um círculo vicioso. ${ }^{71}$

\section{Redução do fluxo sanguíneo}

A redução do fluxo sanguíneo para a musculatura esquelética poderia contribuir para os prejuízos observados nesse tecido devido a uma menor eficiência no transporte de oxigênio, nutrientes, hormônios e metabólitos. Apesar de alguns trabalhos na literatura mostrarem essa redução, ${ }^{49,72,73}$ outros estudos não verificaram essa diminuição, ${ }^{74-78}$ de modo que fatores ainda não conhecidos podem ser determinantes na redução ou manutenção do fluxo sanguíneo.

Além disso, como já foi apresentado, o aumento no débito cardíaco agudo provocado por drogas não melhora a tolerância aos esforços físicos na insufici- ência cardíaca, ${ }^{18,19}$ o que tende a desvalorizar a importância da redução do fluxo sanguíneo como causa das alterações musculares na síndrome.

\section{Tratamento da musculatura esque- lética na insuficiência cardíaca}

\section{Tratamento atual}

Reabilitação cardíaca consiste em exercícios físicos, suporte psicossocial e educação com o propósito de facilitar a readaptação do indivíduo portador de doença cardíaca a uma vida normal, por meio de incrementos em sua capacidade funcional máxima e de reduções de seus fatores de risco para novos eventos cardiovasculares. Os programas e as recomendações da Organização Mundial da Saúde, existentes desde 1964, expandiram o desenvolvimento da reabilitação cardíaca e naquele tempo os primeiros estudos clínicos foram publicados. A reabilitação cardíaca surgiu com programas de exercícios físicos baseados em caminhadas progressivas após infarto do miocárdio e em 1980 tornou uma terapia padronizada nos pacientes internados em hospitais, contrário ao que ocorria antes da década de 1980, quando o tratamento para todos os estágios da insuficiência cardíaca aconselhava o repouso como a primeira linha de defesa destinada a reduzir o estresse sobre o sistema cardiovascular comprometido. Após pouco tempo, testes com exercícios físicos passaram a ser utilizados nesses pacientes e a reabilitação cardíaca tornou parte da vida deles fora do hospital. Metanálises têm sugerido que a reabilitação cardíaca reduz morte total e por causa cardiovascular em cerca de 20 a $25 \%$ e morte súbita em cerca de $37 \%$ durante um ano após um infarto agudo do miocárdio. ${ }^{79,80}$

Está bem estabelecido que o treinamento físico melhora a função muscular em indivíduos normais. $\mathrm{Na}$ insuficiência cardíaca também há fortes indícios de tal melhora, na medida em que estudos mostram melhora na vasodilatação mediada pelo endotélio, na circulação periférica e coronariana e no metabolismo do músculo esquelético. ${ }^{81-84} \mathrm{O}$ treinamento físico também pode reverter muitas das características da miopatia esquelética da insuficiência cardíaca, como mudança no tipo de fibra muscular, atrofia, apoptose e vasoconstrição. ${ }^{85}$ Witham et al. $(2003)^{86}$ mostraram ainda melhora na capacidade de realização de atividade física, diminuição dos sintomas e na atividade dos mecanorreceptores, redução na atividade nervosa simpática concomitante ao aumento na parassimpática, 
decréscimo na expressão de iNOS e citocinas próinflamatórias e, na musculatura esquelética, aumento na capacidade de geração de força, na área de secção transversa da fibra, na tolerância à fadiga, no número de mitocôndrias, no número de capilares por fibra e na atividade de inúmeras enzimas importantes ao metabolismo.

Muitos estudos que investigam os efeitos moleculares do treinamento físico na musculatura esquelética em modelos experimentais e pacientes com insuficiência cardíaca começaram a ser desenvolvidos há pouco tempo, porém os resultados são promissores. ${ }^{87,88}$

Nosso grupo tem evidenciado, por exemplo, que uma melhora funcional na musculatura esquelética de camundongos com insuficiência cardíaca promovida por treinamento físico aeróbico de intensidade moderada, 5 vezes por semana, 60 minutos por sessão e 2 meses de duração, ${ }^{89}$ é acompanhada por aumento na ingestão alimentar e na expressão de proteínas importantes no transporte intracelular de $\mathrm{Ca}^{2+}$ (dados não publicados).

O exercício físico também proporciona a ativação da cascata de sinalização da via AKT/mTOR, iniciada pelo IGF-1, sendo este estímulo um potencial agente trófico para o musculatura esquelética na síndrome. ${ }^{90}$

Outro benefício do treinamento físico é a capacidade de aumentar o sistema antioxidante em pacientes com insuficiência cardíaca. Isso foi demonstrado por um estudo no qual os indivíduos pedalaram em bicicleta ergométrica por seis meses a $70 \%$ do consumo de oxigênio de pico - a atividade da catalase e da glutationa peroxidase foi aumentada concomitantemente à redução na produção de nitrotirosina. ${ }^{91}$ Nesse sentido, outros estudos em pacientes e modelos experimentais de insuficiência cardíaca mostraram que o treinamento físico aeróbio aumentou o sistema antioxidante de 40 a $100 \%$ e reduziu tanto a peroxidação lipídica com a geração de nitrotirosina - além disso, essa redução do estresse oxidativo foi correlacionada com a diminuição da degradação muscular por apoptose. ${ }^{38,65,92,93}$

Além disso, pacientes submetidos a apenas quatro semanas de treinamento físico aeróbio tiveram como benefício uma redução na elevada expressão de MURF-1, uma proteína fundamental no sistema de degradação protéica ubiquitina-proteossoma. ${ }^{67}$

Por fim, é importante ressaltar que todos esses benefícios do exercício físico resultam tanto em melhora na qualidade de vida como aumento na sobrevi- da dos pacientes com insuficiência cardíaca, o que tem sido evidenciado por uma série de estudos. ${ }^{21,94-96}$

Apesar desses benefícios evidenciados na musculatura esquelética dos pacientes com insuficiência cardíaca, dois aspectos devem ser considerados: apesar da importância da musculatura esquelética, descrita anteriormente, ela não recebe tanta atenção como a musculatura cardíaca na terapêutica da síndrome. Além disso, todos esses benefícios foram observados em treinamento físico aeróbio de intensidade moderada, que teoricamente não é o estímulo gera as maiores adaptações no tecido muscular esquelético.

De fato, uma tendência para o futuro em relação ao tipo de exercício físico para pacientes com insuficiência cardíaca provavelmente será a combinação de exercícios voltados à melhora na capacidade aeróbica com exercícios de resistência muscular. ${ }^{24} \mathrm{No}$ estudo de Beckers et al., ${ }^{24}$ por exemplo, nove exercícios de resistência muscular foram executados entre 50 e $60 \%$ do valor de uma repetição máxima, 10 ou 15 repetições, uma ou duas séries, com intervalo de um minuto entre as séries.

\section{Perspectivas futuras}

A terapia padrão da síndrome atualmente visa bloquear os efeitos tóxicos da hiperestimulação do sistema nervoso simpático (antagonistas $\beta$-adrenérgicos) e do sistema renina-angiotensina-aldosterona (inibidores da enzima conversora de angiotensina I e bloqueadores dos receptores de angiotensina $\mathrm{II} \mathrm{AT}_{1}$ ) sobre o tecido cardíaco, ${ }^{97,98}$ porém quase nada é conhecido sobre os efeitos dessas drogas na musculatura esquelética, que inclusive podem ser maléficos.

O treinamento físico aeróbio de moderada intensidade é a única terapia não farmacológica que tem sido utilizado com relativa frequência nessa doença. Estratégias que utilizam exercícios aeróbios intervalados de maior intensidade e, principalmente, treinamentos de força, deveriam ser implementados com maior frequência nesses pacientes com o objetivo de terem suas eficácias testadas no tratamento das alterações da musculatura esquelética características da síndrome.

Outra terapia não farmacológica que não tem recebido a devida atenção é o acompanhamento nutricional. Como vimos, a menor ingestão energética e de aminoácidos, associadas à má absorção intestinal, são traços clássicos da síndrome. ${ }^{52}$ Com o objetivo de avaliar as consequências dessa menor ingestão alimentar, estudamos um grupo de animais controles que ingeriram a mesma quantidade de ração dos animais 
com insuficiência cardíaca, o que correspondeu a uma restrição de $14 \%$. Interessantemente, esses animais apresentaram prejuízos equivalentes na geração de força e na expressão de proteínas que transportam o $\mathrm{Ca}^{2+}$ intracelular quando comparados aos animais com insuficiência cardíaca (dados não publicados), o que corrobora a importância de intervenções nutricionais detalhadas no tratamento da síndrome. De fato, uma série de investigações científicas tem demonstrado que diferentes suplementações nutricionais (dieta hiperprotéica, aminoácidos, creatina e micronutrientes) geram uma série de efeitos benéficos em pacientes com insuficiência cardíaca, inclusive melhora na qualidade de vida. ${ }^{99-102}$

Por fim, é importante ressaltar que começam a aparecer na literatura resultados promissores em modelos animais e pacientes submetidos a terapias celulares em relação ao tecido muscular cardíaco na insuficiência cardíaca. ${ }^{103}$ No entanto, devido à importância da musculatura esquelética na síndrome, discutida no início do presente estudo, em breve terapias celulares e gênicas provavelmente começarão a ser testadas com o objetivo de reverter a deterioração desse tecido. Em relação à terapia gênica, potencialmente várias manipulações genéticas podem ser benéficas, como das proteínas que transportam o $\mathrm{Ca}^{2+}$, participam dos processos de síntese e degradação protéicas, e regulam a angiogênese. A Figura 5 suma- riza as principais formas de tratamento para a insuficiência cardíaca.

\section{Considerações finais}

Em pacientes com insuficiência cardíaca, conforme foi discutido, o grau de deterioração do tecido muscular esquelético tem relação direta com a qualidade de vida e autonomia, por meio do nível de tolerância aos esforços físicos, além de ser um preditor independente de mortalidade na síndrome.

Portanto, a musculatura esquelética deveria merecer atenção considerável no tratamento da insuficiência cardíaca. No entanto, isso não ocorre, pois o efeito da terapia farmacológica padrão dos pacientes na musculatura esquelética não é conhecido e apenas os efeitos do treinamento físico aeróbio de moderada intensidade são consideravelmente elucidados nesse tecido.

Além de drogas, exercícios físicos aeróbios de maior intensidade e intervalados, exercícios físicos de força, intervenções nutricionais e terapias celulares e gênicas são linhas de pesquisa pouco ou nada exploradas que potencialmente podem regredir a degeneração no tecido muscular esquelético dos pacientes e, consequentemente, contribuir para melhorar a qualidade de vida, a autonomia e o prognóstico da insuficiência cardíaca.

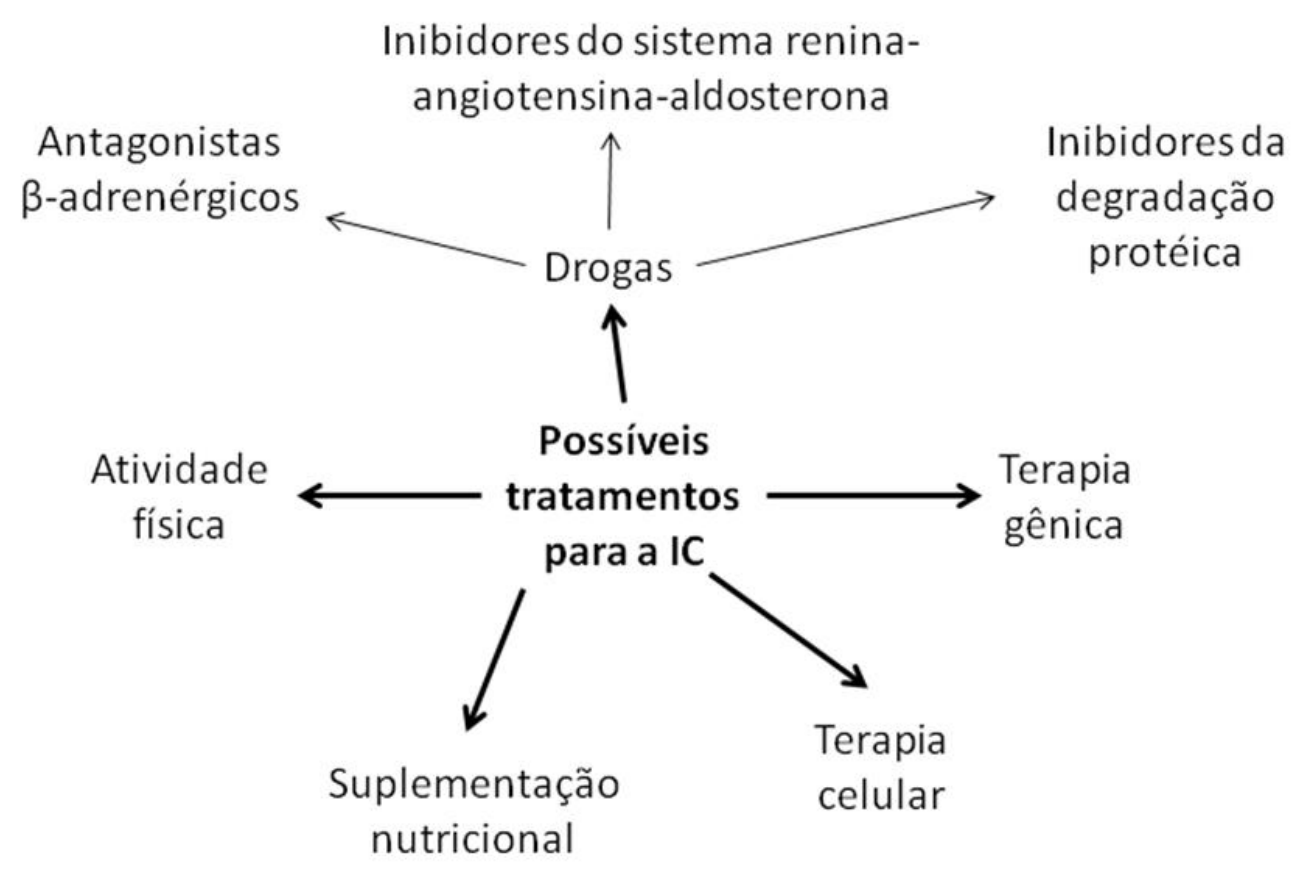

Figura 5: Possíveis tratamentos para a insuficiência cardíaca (IC), incluindo os atuais e as perspectivas futuras. 


\begin{abstract}
Heart failure patients, the final pathway of the cardiovascular diseases and the first cause of admission in hospital in Brazil, present skeletal musculature injuries. Furthermore, the damages in this musculature have direct relationship with the quality of life and the autonomy of the patients, by the physical exercise tolerance, and are an independent predictor of mortality in the syndrome.

During this review will be discussed about heart failure: its concept and a brief history; the importance of the skeletal musculature; the functional, structural, biochemical and molecular injuries that undergoes this tissue; the main causes of these damages (sympathetic hyperactivity, decreased food intake, reactive oxygen species, inflammatory cytokines and reduced physical activity level and blood flow); and the effects of the principal therapy to prevent this dysfunction, the moderated intensity aerobic physical training.

If we agree about the importance of the skeletal muscle tissue in the syndrome, this is an area of scientific investigation with many subjects still not studied, like the effect of: pharmacological therapies used by the patients, high intensity aerobic physical exercises, strength physical exercises, nutritional interventions and cellular and genetic therapies. In the future, the results of these studies that will be developed certainly will contribute to less degeneration of the skeletal musculature in the disease, what, consequently, will improve the quality of life, the autonomy and the prognosis of the patients.
\end{abstract}

Keywords: Heart Failure. Musculoskeletal System, Molecular Biology. Therapeutics.

\section{Referências bibliográficas}

1. Prata PR. Uneven development and the inequaliy of mortality in Brazil. [Tese de mestrado], Leeds: Department of Public Health Medicine - Leeds University, 1989.

2. Araújo DV, Tavares LR, Veríssimo R, Ferraz MB, Mesquita ET. Custo da insuficiência cardíaca no Sistema Único de Saúde. Arq Bras Cardiol. 2005; 84: 422-7.

3. Davis RC, Hobbs FDR, Lip GYH. ABC of heart failure: history and epidemiology. Br Med J. 2000; 320:39-42.

4. Marks AR. A guide for the perplexed towards in understanding of the molecular basis of heart failure. Circulation. 2003; 107:1456-9.

5. Colucci WS. The effects of norepinephrine on myocardial biology: implications for the therapy of heart failure. Cardiol Clín. 1998; 21:120-4.

6. Lunde PK, Sjaastad I, Schiøtz Thorud HM, Sejersted OM. Skeletal muscle disorders in heart failure. Acta Physiol Scand. 2001; 171:277-94.

7. Cohn JN, Ferrari R, Sharpe N. Cardiac remodeling - concepts and clinical implications: a consensus paper from the International Forum on cardiac remodeling. J Am Coll Cardiol. 2000; 35:569-82.

8. Ferrari R, Ceconi C. Neuroendocrine activation in left ventricular dysfunction. Eur Heart J. 1998; 19:1423-4, 1998.

9. Drexler H, Hayoz D, Münzel T, Hornig B, Just H, Brunner HR et al. Endothelial function in chronic congestive heart failure. Am J Cardiol. 1992; 69:1596-601.

10. Anker SD, Chua TP, Ponikowski P, Harrington D, Swan JW, Kox WJ et al. Hormonal changes and catabolic/anabolic imbalance in chronic heart failure and their importance for cardiac cachexia. Circulation.. 1997; 92:526-34.

11. Schulze PC, Gielen S, Schuler G, Hambrecht R. Chronic heart failure and skeletal muscle catabolism: effects of exercise training. Int J Cardiol. 2002; 85:141-9.

12. Morley JE, Thomas DR, Wilson MM. Cachexia: pathophysiology and clinical relevance. Am J Clin Nutr. 2006; 83: 735-43.
13. Anker SD, Swan JW, Volterrani M, Chua TP, Clark AL, PooleWilson PA, Coats AJ. The influence of muscle mass, strength, fatigability and blood flow on exercise capacity in cachectic and non-cachectic patients with chronic heart failure. Eur Heart J. 1997; 18:259-69.

14. Franciosa JA, Park M, Levine TB. Lack of correlation between exercise capacity and indexes of resting left ventricular performance in heart failure. Am J Cardiol. 1981; 47:33-9.

15. Wilson JR, Rayos G, Yeoh TK, Gothard P, Bak K. Dissociation between exertional symptoms and circulatory function in patients with heart failure. Circulation. 1995; 92:47-53.

16. Minotti JR, Christoph I, Oka R, Weiner MW, Wells L, Massie BM. Impaired skeletal muscle function in patients with congestive heart failure: relationship to systemic exercise performance. J Clin Invest. 1991; 88:2077-82.

17. Volterrani M, Clark AL, Ludman PF, Swan JW, Adamapoulos $\mathrm{S}$, Piepoli, $\mathrm{M}$ et al. Predictors of exercise capacity in chronic heart failure. Eur Heart J. 1994; 15:801-9.

18. Wilson JR. Exercise intolerance in heart failure. Circulation. 1995; 91:559-61.

19. Dayer MJ, Hopkinson NS, Ross ET, Jonville S, Sharshar T, Kearney $\mathrm{M}$ et al. Does symptom-limited cycle exercise cause low frequency diaphragm fatigue in patients with heart failure? Eur J Heart Fail. 2006; 8:68-73.

20. Jondeau G, Katz SD, Zohman L, Goldberger M, Mccarthy M, Bourdarias JP et al. Active skeletal muscle mass and cardiopulmonary reserve. Failure to attain peak aerobic capacity during maximal bicycle exercise in patients with severe congestive heart failure. Circulation. 1992; 86:1351-6.

21. Crimi E, Ignarro LJ, Cacciatore F, Napoli C. Mechanisms by which exercise training benefits patients with heart failure. Nat Rev Cardiol. 2009; 6:292-300.

22. Stainsby WN, Sumners C, Eitzman PD. Effects of catecholamines on lactic acid output during progressive working contractions. J Appl Physiol. 1985; 59:1809-14. 
23. Vescovo G, Zennaro R, Sandri M, Carraro U, Leprotti C, Ceconi $\mathrm{C}$ et al. Apoptosis of skeletal muscle myofibers and interstitial cells in experimental heart failure. J Mol Cell Cardiol. 1998; 30:2449-59.

24. Beckers PJ, Denollet J, Possemiers NM, Wuyts FL, Vrints CJ, Conraads VM. Combined endurance-resistance training vs. endurance training in patients with chronic heart failure: a prospective randomized study. Eur Heart J. 2008; 29:185866.

25. Kamalakkannan G, Petrilli CM, George I, LaManca J, McLaughlin BT, Shane E, Mancini DM, Maybaum S. Clenbuterol increases lean muscle mass but not endurance in patients with chronic heart failure. J Heart Lung Transplant. 2008; 27:457-61.

26. Schulze PC, Fang J, Kassik KA, Gannon J, Cupesi M, MacGillivray $C$ et al. Transgenic overexpression of locally acting insulin-like growth factor-1 inhibits ubiquitin-mediated muscle atrophy in chronic left-ventricular dysfunction. Circ Res. 2005; 97:418-26.

27. Ringer S. Concernig the influence exerted by each of the constituents of the blood on the contraction of the ventricle. J Physiol. 1882; 3:380-3.

28. Perreault CL, Gonzalez-Serratos H, Litwin SE, Sun X, FranziniArmstrong $\mathrm{C}$, Morgan JP. Alterations in contractility and intracellular $\mathrm{Ca} 2+$ transients in isolated bundles of skeletal muscle fibers from rats with chronic heart failure. Circ Res.. 1993; 73:405-12.

29. Massie BM, Simonini A, Sahgal P, Wells L, Dudley GA. Relationship of systemic and local muscle exercise capacity to skeletal muscle characteristics in patients with congestive heart failure. J Am Coll Cardiol. 1996; 27:140-6.

30. Buller NP, Jones DA, Poole-Wilson PA. Direct measurement of skeletal muscle fatigue in patients with chronic heart failure. Br Heart J. 1991; 65:20-4.

31. Minotti JR, Pillay P, Chang L, Wells L, Massie BM. Neurophysiological assessment of in patients with congestive heart failure. Circulation. 1992; 86:903-908.

32. Simonini A, Chang K, Yue P, Long CS, Massie BM. Expression of skeletal muscle sarcoplasmic reticulum calcium-ATPase is reduced in rats with postinfarction heart failure. Heart 1999; 81:303-7.

33. Lunde PK, Dahlstedt AJ, Bruton JD, Lännergren J, Thorén P, Sejersted $\mathrm{OM}$ et al. Contraction and intracellular $\mathrm{Ca} 2+$ handling in isolated skeletal muscle of rats with congestive heart failure. Circ Res. 2001; 88:1299-305.

34. Bartoli M, Richard I. Calpains in muscle wasting. Int J Biochem Cell Biol. 2005; 37: 2115-33.

35. Dominguez JF, Howell S. Compartmental analysis of steadystate diaphragm $\mathrm{Ca} 2+$ kinetics in chronic congestive heart failure. Cell Calcium. 2003; 33:163-74.

36. Gomes MD, Lecker SH, Jagoe RT, Navon A, Goldberg AL. Atrogin-1, a muscle-specific F-box protein highly expressed during muscle atrophy. Proc Natl Acad Sci U. S. A. 2001; 98:14440-5.

37. Bodine SC, Stitt TN, Gonzalez M, Kline WO, Stover GL, Bauerlein $R$ et al. Akt/mTOR pathway is a crucial regulator of skeletal muscle hypertrophy and can prevent muscle atrophy in vivo. Nat Cell Biol. 2001; 3:1014-9.

38. Price SR, Bailey JL, Wang X, Jurkovitz C, England BK, Ding X et al. Muscle wasting in insulinopenic rats results from activation of the ATP-dependent, ubiquitin-proteasome proteolytic pathway by a mechanism including gene transcription. J Clin Invest. 98: 1703-8, 1996.
39. Tiao G, Fagan JM, Samuels N, James JH, Hudson K, Lieberman $M$ et al. Sepsis stimulates nonlysosomal, energy-dependent proteolysis and increases ubiquitin mRNA levels in rat skeletal muscle. J Clin Invest. 1994; 94: 2255-64.

40. Dalla Libera L, Ravara B, Volterrani M, Gobbo V, Della Barbera $\mathrm{M}$, Angelini A et al. Beneficial effects of GH/IGF-1 on skeletal muscle atrophy and function in experimental heart failure. Am J Physiol Cell Physiol. 2004; 286:138-44.

41. Yimlamai T, Dodd SL, Borst SE, Park S. Clenbuterol induces muscle-specific attenuation of atrophy through effects on the ubiquitin-proteasome pathway. J Appl Physiol. 2005; 99: 71-80.

42. Higgins JA, Lasslett YV, Bardsley RG, Buttery PJ. The relation between dietary restriction or clenbuterol (a selective beta 2 agonist) treatment on muscle growth and calpain proteinase (EC 3.4.22.17) and calpastatin activities in lambs. Br J Nutr. 1998; 60:645-52.

43. Navegantes LC, Resano NM, Baviera AM, Migliorini RH, Kettelhut C. Effect of sympathetic denervation on the rate of protein synthesis in rat skeletal muscle. Am J Physiol. 2004; 286:642-7.

44. Kim YS, Lee YB, Ashmore CR. Cimaterol-induced growth in rats: growth pattern and biochemical characteristics. Growth Dev Aging. 1988; 52: 41-5.

45. Kim YS, Lee YB, Garrett WN, Dalrymple RH. Effects of cimaterol on nitrogen retention and energy utilization in lambs. J Anim Sci. 1989; 67:674-81.

46. Mcelligott MA, Barreto AJR, Chaung LY. Effect of continuous and intermittent clenbuterol feeding on rat growth rate and muscle. Comp Biochem Physiol. 1989; 92:135-8.

47. Rothwell NJ, Stock MJ, Sudera DK. Changes in tissue blood flow and beta-receptor density of skeletal muscle in rats treated with the beta2-adrenoceptor agonist clenbuterol. $\mathrm{Br}$ J Pharmacol. 1987; 90:601-7.

48. Jensen J, Brennesvik EO, Bergersen $H$, Oseland $H$, Jebens E, Brors O. Quantitative determination of cell surface betaadrenoceptors in different rat skeletal muscles. Pflugers arch., Eur J Physiol. 2002; 444:213-9.

49. Negrão CE, Brandão Rondon MUP, Tinucci T, Alves MJN, Roveda F, Braga ANW et al. Abnormal neurovascular control during exercise is linked to heart failure severity. Am J Physiol, Heart Circ Physiol. 2001; 280:1286-92.

50. Schwengel RH, Gottlieb SS, Fisher ML. Protein-energy malnutrition in patients with ischemic and nonischemic dilated cardiomyopathy and congestive heart failure. Am J Cardiol. 1994; 73:908-10.

51. Lennie TA, Moser DK, Heo S, Chung ML, Zambroski CH. Factors influencing food intake in patients with heart failure. $J$ Cardiovasc Nurs. 2006; 21:123-9.

52. Franssen FME, Wouters EFM, Schols AMWJ. The contribution of starvation, deconditioning and ageing to the observed alterations in peripheral skeletal muscle in chronic organ diseases. Clin Nutr. 2002; 21:1-14.

53. Linden CGVD, Simonides WS, Muller A, Laarse WJVD, Vermeulen JL, Zuidwijk MJ et al. Fiber-specific regulation of $\mathrm{Ca}(2+)$-ATPase isoform expression by thyroid hormone in rat skeletal muscle. Am J Physiol. 1996; 271:1908-19.

54. Reid MB. Invited Review: redox modulation of skeletal muscle contraction: what we know and what we don't. J Appl Physiol. 2001; 90:724-31. 
55. Kondo H, Miura M, Itokawa $\mathrm{Y}$. Oxidative stress in skeleta muscle atrophied by immobilization. Acta Physiol Scand. 1991; 142:527-8.

56. Lawler JM, Song W, Demaree SR. Hindlimb unloading increases oxidative stress and disrupts antioxidant capacity in skeletal muscle. Free Radic Biol Med. 2003; 35:9-16.

57. Appell HJ, Duarte JA, Soares JM. Supplementation of vitamin E may attenuate skeletal muscle immobilization atrophy. Int $J$ Sports Med. 1997; 18:157-60.

58. Betters JL, Criswell DS, Shanely RA, Van Gammeren D, Falk $\mathrm{D}$, Deruisseau KC et al. Trolox attenuates mechanical ventilation-induced diaphragmatic dysfunction and proteolysis. Am J Respir Crit Care Med. 2004; 170:1179-84.

59. Li YP, Chen Y, Li AS, Reid MB. Hydrogen peroxide stimulates ubiquitin-conjugating activity and expression of genes for specific E2 and E3 proteins in skeletal muscle myotubes. Am J Physiol Cell Physiol. 2003; 285:806-12.

60. Wyke SM, Russell ST, Tisdale MJ. Induction of proteasome expression in skeletal muscle is attenuated by inhibitors of NF-kappaB activation. Br J Cancer. 2004; 91:1742-50.

61. Gielen S, Adams V, Linke A, Erbs S, Möbius-Winkler S, Schubert A, Schuler G, Hambrecht R. Exercise training in chronic heart failure: correlation between reduced local inflammation and improved oxidative capacity in the skeletal muscle. Eur J Cardiovasc Prev Rehabil. 2005; 12:393-400.

62. Linke A, Adams V, Schulze PC, Erbs S, Gielen S, Fiehn E, Möbius-Winkler S, Schubert A, Schuler G, Hambrecht R. Antioxidative effects of exercise training in patients with chronic heart failure: increase in radical scavenger enzyme activity in skeletal muscle. Circulation. 2005; 111:1763-70.

63. Feldman AM, Combes A, Wagner D, Kadakomi T, Kubota T, Li YY et al. The role of tumor necrosis factor in the pathophysiology of heart failure. J Am Coll Cardiol. 2000; 35:537-44.

64. McTiernan CF, Lemster BH, Frye C, Brooks S, Combes A, Feldman AM. Interleukin-1 beta inhibits phospholamban gene expression in cultured cardiomyocytes. Circ Res. 1997; 81:493-503.

65. Adams A, Spate U, Krandel N, Schulze PC, Linke A, Schuler G et al. Nuclear factor-kappa B activation in skeletal muscle of patients with chronic heart failure: correlation with the expression of inducible nitric oxide synthase. Eur J Cardiovasc Prev Rehabil. 2003; 10:273-7.

66. Adams V, Nehrhoff B, Späte U, Linke A, Schulze PC, Baur A et al. Induction of iNOS expression in skeletal muscle by IL1 beta and NFkappaB activation: an in vitro and in vivo study. Cardiovasc Res. 2002; 54:95-104.

67. Gielen S, Adams V, Mobius-Winkler S, Linke A, Erbs S, Yu J et al. Anti-inflammatory effects of exercise training in the skeletal muscle of patients with chronic heart failure. J Am Coll Cardiol. 2003; 42: 861-8.

68. Paulus WJ. How are cytokines activated? Eur J Heart Fail., 1999; 1: 309-12.

69. Hambrecht R, Adams V, Gielen S, Linke A, Möbius-Winkler S, $\mathrm{Yu} \mathrm{J}$ et al. Exercise intolerance in patients with chronic heart failure and increased expression of inducible nitric oxide synthase in the skeletal muscle. J Am Coll Cardiol. 1999; 33:174-9.

70. Hambrecht R, Schulze PC, Gielen S, Linke A, Mobius-Winkler $\mathrm{S}, \mathrm{Yu} \mathrm{J}$ et al. Reduction of insulin-like growth factor-I expression in the skeletal muscle of noncachectic patients with chronic heart failure. J Am Coll Cardiol. 2002; 39:1175-81.
71. Winter MM, Bouma BJ, van Dijk AP, Groenink M, Nieuwkerk PT, van der Plas MN et al. Relation of physical activity, cardiac function, exercise capacity, and quality of life in patients with a systemic right ventricle. Am J Cardiol. 2008; 102:125862.

72. Wilson JR, Martin JL, Schwartz D, Ferraro N. Exercise intolerance in patients with chronic heart failure: role of impaired nutritive flow to skeletal muscle. Circulation. 1984; 69:107987.

73. Sullivan MJ, Knight JD, Higginbotham MB, Cobb FR. Relation between central and peripheral hemodynamics during exercise in patients with chronic heart failure. Muscle blood flow is reduced with maintenance of arterial perfusion pressure. Circulation. 1989; 80:769-81.

74. Wilson JR, Fink L, Maris J, Ferraro N, Power-Vanwart J, Eleff $S$ et al. Evaluation of energy metabolism in skeletal muscle of patients with heart failure with gated phosphorus-31 nuclear magnetic resonance. Circulation. 1985; 71:57-62.

75. Wiener DH, Fink LI, Maris J, Jones RA, Chance B, Wilson JR. Abnormal skeletal muscle bioenergetics during exercise in patients with heart failure: role of reduced muscle blood flow. Circulation. 1986; 73:1127-36.

76. Mancini DM, Ferraro N, TUchler M, Chance B, Wilson JR. Detection of abnormal calf muscle metabolism in patients with heart failure using phosphorus-31 nuclear magnetic resonance. Am J Cardiol. 1988; 62:1234-40.

77. Mancini DM, Coyle E, Coggan A, Beltz J, Ferraro N, Montain S et al. Contribution of intrinsic skeletal muscle changes to 31P NMR skeletal muscle metabolic abnormalities in patients with chronic heart failure. Circulation. 1989; 80:1338-46.

78. Massie B, Conway M, Yonge R, Frostick S, Ledingham J, Sleight $\mathrm{P}$ et al. Skeletal muscle metabolism in patients with congestive heart failure: relation to clinical severity and blood flow. Circulation. 1987; 76:1009-19.

79. Velasco Rami JA, Maroto Montero JM. Cardiac rehabilitation its historical evolution and current situation. Rev Esp Cardiol. 1995; 48:1-7.

80. Pashkow FJ. Issues in contemporary cardiac rehabilitation: a historical perspective. J Am Coll Cardiol. 1993; 21:822-34.

81. Negrão CE, Middlekauff HR. Exercise training in heart failure: reduction in angiotensin II, sympathetic nerve activity, and baroreflex control. J Appl Physiol. 2008; 104(3):577-8.

82. Adamopoulos S, Parissis J, Karatzas D, Kroupis C, Georgiadis $M$, Karavolias $G$ et al. Physical training modulates proinflammatory cytokines and the soluble Fas/soluble Fas ligand system in patients with chronic heart failure. J Am Coll Cardiol. 2002; 39:653-63

83. Hornig B, Maier V, Drexler H. Physical training improves endothelial function in patients with chronic heart failure. Circulation. 1996; 93:210-14.

84. Wang J, Yi GH, Knecht M, Cai BL, Poposkis S, Packer M et al. Physical training alters the pathogenesis of pacing-induced heart failure through endothelium-mediated mechanisms in awake dogs. Circulation. 1997; 96:2683-92.

85. Clark AL, Poole-Wilson PA, Coats AJ. Exercise limitation in chronic heart failure: central role of the periphery. J Am Coll Cardiol. 1996; 28:1092-102.

86. Witham MD, Struthers AD, Mcmurdo ME. Exercise training as a therapy for chronic heart failure: can older people benefit? J Am Geriatr Soc. 2003; 51:699-709. 
87. Bacurau AV, Jardim MA, Ferreira JC, Bechara LR, Bueno CR Jr, Alba-Loureiro TC, Negrao CE, Casarini DE, Curi R, Ramires PR, Moriscot AS, Brum PC. Sympathetic hyperactivity differentially affects skeletal muscle mass in developing heart failure: role of exercise training. J Appl Physiol. 2009; 106:1631-40.

88. Papathanasiou G, Tsamis N, Georgiadou P, Adamopoulos S. Beneficial effects of physical training and methodology of exercise prescription in patients with heart failure. Hell $\mathrm{J}$ Cardiol. . 2008; 49:267-77.

89. Ferreira JC, Rolim NP, Bartholomeu JB, Gobatto CA, Kokubun E, Brum PC. Maximal lactate steady state in running mice: effect of exercise training. Clin Exp Pharmacol Physiol. 2007; 34:760-5.

90. Mascher $\mathrm{H}$, Andersson $\mathrm{H}$, Nilsson PA, Ekblom B, Blomstrand E. Changes in signalling pathways regulating protein synthesis in human muscle in the recovery period after endurance exercise. Acta physiol (Oxf) 2007; 191:65-75

91. Linke A, Adams V, Schulze PC, Erbs S, Gielen S, Fiehn E et al. Antioxidative effects of exercise training in patients with chronic heart failure: increase in radical scavenger enzyme activity in skeletal muscle. Circulation. 2005; 111:1763-70.

92. Medina R, Wing SS, Goldberg AL. Increase in levels of polyubiquitin and proteasome mRNA in skeletal muscle during starvation and denervation atrophy. Biochem J. 1995; 307:631-7.

93. Mitch WE, Medina R, Grieber S, May RC, England BK, Price SR et al. Metabolic acidosis stimulates muscle protein degradation by activating the adenosine triphosphate-dependent pathway involving ubiquitin and proteasomes. J Clin Invest. 1994; 93:2127-33.

94. Patwala AY, Woods PR, Sharp L, Goldspink DF, Tan LB, Wright DJ. Maximizing patient benefit from cardiac resynchronization therapy with the addition of structured exercise training: a randomized controlled study. J Am Coll Cardiol. 2009; 53:23329.

95. Belardinelli R, Georgiou D, Cianci G, Purcaro A. Exercise training for patients with chronic heart failure reduced mortality and cardiac events and improved quality of life. West $J$ Med. 2000; 172:28.
96. O'Connor CM, Whellan DJ, Lee KL, Keteyian SJ, Cooper LS, Ellis SJ, Leifer ES, Kraus WE, Kitzman DW, Blumenthal JA, Rendall DS, Miller NH, Fleg JL, Schulman KA, McKelvie RS, Zannad F, Piña IL; HF-ACTION Investigators. Efficacy and safety of exercise training in patients with chronic heart failure: HF-ACTION randomized controlled trial. JAMA 2009; 301:1439-50.

97. Katz AM. Potential deleterious effects of inotropic agents in the therapy of chronic heart failure. Circulation. 1986; 73:184-90.

98. Packer M. Treatment of chronic heart failure. Lancet 1992; 340:92-5.

99. Evangelista LS, Heber D, Li Z, Bowerman S, Hamilton MA, Fonarow GC. Reduced body weight and adiposity with a high-protein diet improves functional status, lipid profiles, glycemic control, and quality of life in patients with heart failure: a feasibility study. J Cardiovasc Nurs. 2009; 24:20715.

100. Scognamiglio R, Testa A, Aquilani R, Dioguardi FS, Pasini E. Impairment in walking capacity and myocardial function in the elderly: is there a role for nonpharmacologic therapy with nutritional amino acid supplements? Am J Cardiol. 2008; 101:78E-81E.

101. Kuethe F, Krack A, Richartz BM, Figulla HR. Creatine supplementation improves muscle strength in patients with congestive heart failure. Pharmazie 2006; 61:218-22.

102. Witte KK, Nikitin NP, Parker AC, von Haehling S, Volk HD, Anker SD, Clark AL, Cleland JG. The effect of micronutrient supplementation on quality-of-life and left ventricular function in elderly patients with chronic heart failure. Eur Heart J. 2005; 26:2238-44.

103. Becker C, Lacchini S, Muotri AR, Silva GJ, Castelli JB, Vassallo PF et al. Skeletal muscle cells expressing VEGF induce capillary formation and reduce injury in rats. Int J Cardiol. 2006; 39:89-97. 